Article

\title{
Statistical Error for Cosmic Rays Modulation Evaluated by SDE Backward in Time Method for 1D Model
}

\author{
Viacheslav Mykhailenko ${ }^{1, *,+(1)}$ and Pavol Bobik ${ }^{2,+}+($ ) \\ 1 Institute of Physics, Faculty of Science, P. J. Safarik University, Park Angelinum 9, 04154 Košice, Slovakia \\ 2 Institute of Experimental Physics, Slovak Academy of Sciences, Watsonova 47, 04001 Košice, Slovakia; \\ bobik@saske.sk \\ * Correspondence: viacheslav.mykhailenko@student.upjs.sk \\ + These authors contributed equally to this work.
}

check for updates

Citation: Mykhailenko, V.; Bobik, P. Statistical Error for Cosmic Rays Modulation Evaluated by SDE Backward in Time Method for 1D Model. Fluids 2022, 7, 46. https:// doi.org/10.3390/fluids7020046

Academic Editors: Gang Li and Mehrdad Massoudi

Received: 15 November 2021

Accepted: 14 January 2022

Published: 19 January 2022

Publisher's Note: MDPI stays neutral with regard to jurisdictional claims in published maps and institutional affiliations.

Copyright: (c) 2022 by the authors. Licensee MDPI, Basel, Switzerland. This article is an open access article distributed under the terms and conditions of the Creative Commons Attribution (CC BY) license (https:// creativecommons.org/licenses/by/ $4.0 /)$.

\begin{abstract}
The propagation of cosmic rays through the heliosphere has been solved for more than half a century by stochastic methods based on Ito's lemma. This work presents the estimation of statistical error of solution of Fokker-Planck equation by the 1D backward in time stochastic differential equations method. The error dependence on simulation statistics and energy is presented for different combinations of input parameters. The $1 \%$ precision criterion in mean value units of intensity standard deviation is defined as a function of solar wind velocity and diffusion coefficient value.
\end{abstract}

Keywords: cosmic rays; heliosphere; Parker equation

\section{Introduction}

The Sun produces and radiates out a charged particle flow, which is called the solar wind. The solar wind propagates through the solar system and continues to the moment when the solar wind and the interstellar wind pressure are balanced. This spherical region, which has a radius that is equal approximately $100 \mathrm{AU}$, is called the heliosphere. The socalled solar modulation process begins when the galactic cosmic rays (GCRs) reach the heliosphere's boundary, which presents a decrease of GCR intensity inside the heliosphere (mostly for particles with energies less than $30 \mathrm{GeV}$ ). At the time of solar modulation, GCR particles interact with magnetic irregularities in the solar wind. This process can be approximated as a diffusion combined with convection and adiabatic energy losses. The particle randomly walking between these irregularities. These irregularities move with the velocity of the solar wind. Consequently, the GCR's intensity is strongly anticorrelated concerning solar activity, and is also influenced by the interplanetary magnetic field polarity and GCR particle charge sign. Parker [1] introduced a widely-used equation to describe GCR propagation inside the heliosphere. One of the most precise methods to solve this equation is the so-called stochastic integration method. Using this approach, we can evaluate the solution calculated as the associated set of stochastic differential equations (SDEs), "forward-in-time" or "backward-in-time". The stochastic approach to solving the Parker transport equation is based on Ito's lemma; however, first forward-in-time stochastic solutions as [2] were based on [3] (Equation (223)). Backward-in-time was introduced in article [4], and precisely described, for example, in [5]. The backward-in-time SDE method was widely applied in studying galactic cosmic rays modulation [6-14]. SDE method is very nicely and deeply explained in Hitch-hiker's Guide to Stochastic Differential Equations $[15,16]$.

This article used the backward-in-time stochastic integration approach for all of the simulations. In this approach, quasi-particle objects were injected at the registration boundary inside the heliosphere. They then move back in time through the heliosphere until they reached the heliosphere outer boundary. Both forward and backward approaches descend from the Kolmogorov forward and Kolmogorov backward equations, which is the reason 
why they have the same mathematical description. Results of both methods are compared in [16-18].

The problem of cosmic ray modulation is intrinsically a time-dependent problem. Hysteresis effect appears in cosmic rays intensities and solar parameters dependencies, as sunspot numbers, $10.7 \mathrm{~cm}$ solar flux, and other solar parameters.

In this paper, we evaluate the solution of the Parker equation using the backward-intime stochastic integration approach. We focus on the estimation of statistical error for 1D backward- in-time stochastic differential equations method. Preliminary analysis of statistical error for 1D forward-in-time method we present in article [19]. Scaling study of the SDE approach application to cosmic ray modulations showing the influence of a different number of injected particles on realistic test problem is presented in [20]. In this article, we present an explanation of how statistical error in the backward SDE method appears and show how someone with his own SDE code, could fastly estimate the dependence of statistical error of his own method on a number of injected particles. Additionally, we focus on the development of a method allowing a scan of the parameter space of the model to evaluate statistical error dependence on the model's parameters.

For the sake of clarity, this article has focused on the solution of the 1D Parker equation. The solutions that are presented were evaluated for different sets of input parameters. The injection energy range was taken to be from $1 \mathrm{GeV}$ up to $100 \mathrm{GeV}$.

\section{Model Description}

In 1965, Parker proposed a transport equation that describes the GCR distribution inside the heliosphere [1]. The equation that describes the solar modulation and particle propagation process in the heliosphere can be written through an omnidirectional distribution function $f(\vec{x}, p)$, with particle momentum $p$ in the following form:

$$
\frac{\partial f}{\partial t}=-\nabla \cdot(f \vec{V})+\nabla \cdot[\tilde{K} \cdot \nabla f]+\frac{\nabla \cdot \vec{V}}{3 p^{2}} \frac{\partial}{\partial p}\left(p^{3} f\right)
$$

Here $V=V_{s w}+V_{d r i f t}, V_{s w}$ is solar wind velocity, $V_{d r i f t}$ is the particle magnetic drift velocity, $\vec{x}$ is the $3-\mathrm{D}$ spatial position in Cartesian coordinates, and $\tilde{K}$ is the diffusion tensor. The differential intensity $J$ is related to $f$ as $J=p^{2} f$.

The stochastic SDE set integrations should be performed in a Euclidean space. The set of the spatial position could be pronounced as a set of Cartesian coordinates [5]. In 2010, Pei et al. found that spherical coordinates can be successfully applied [21]. For 1D representation of the heliosphere in spherical coordinates, and for the case where all parameters depend only on radius and energy, the Parker equations can be simplified to the following form $[17,22]$ :

$$
\frac{\partial f}{\partial t}=\frac{1}{r^{2}} \frac{\partial}{\partial r}\left(r^{2} K \frac{\partial}{\partial r} f\right)-\frac{1}{r^{2}} \frac{\partial r^{2} V f}{\partial r}+\frac{1}{3}\left(\frac{1}{r^{2}} \frac{\partial r^{2} V}{\partial r}\right) \frac{1}{p^{2}} \frac{\partial}{\partial p}\left(p^{3} f\right)
$$

Where the diffusion tensor $\tilde{K}$ was simplified to be a scalar $K=K_{0} \beta P$, here $K_{0}$ is the diffusion parameter, $\beta$ is the particle velocity in speed of light units, and $P$ is the rigidity in gigavolt units. Given that the magnetic field is assumed to be spherically symmetrical in $1 \mathrm{D}$ representation, the magnetic drift velocity in the radial direction is equal to zero. Then drift velocity present in the $3 \mathrm{D}$ representation has zero value in the $1 \mathrm{D}$ representation. The main difference between 1D and 2D or 3D models is the presence of drift in 2D and 3D models. Particle drift caused by the large-scale heliospheric magnetic field is evaluated from the anti-symmetric component of the diffusion tensor $[2,23,24]$. Comparison of 1D, 2D, and 3D models could be found in [21]. 


\section{Forward-in-Time Stochastic Integrations}

The stochastic path of a particle in the forward-in-time approach is described by SDE (12) (13) and (14) in [17]. In this approach, a quasi-particle injected at the heliosphere's border (for all of the simulations presented in this work) is spherical with a radius of $100 \mathrm{AU}$ and it does not have any structure (i.e., termination shock, heliosheath, heliopause, or bow shock). For each step of time, the particle lost its energy (momentum) by the value calculated in Equation (13). Every time that the particle crosses a 1 AU registration radius, its actual energy and position are registered to an appropriated energy bin. The position at $0.01 \mathrm{AU}$ was set as an inner reflecting boundary in all of the presented simulations (so-called mirroring). As pointed out by [21], the quasi-particle object is not a real particle but is simply a point in phase space.

\section{Backward-in-Time Stochastic Integrations}

The modulated spectra at $1 \mathrm{AU}$ position were evaluated using the procedure described in [17] with LIS from [22], and thus the differential intensity was taken to be $J \propto p\left(m^{2} c^{4}+p^{2} c^{2}\right)^{-1.85}$. The time step for all of the backward-in-time stochastic integrations that are presented in this article is constant and taken to be $\Delta t=5 \mathrm{~s}$. In the presented article, all backward-in-time stochastic integrations are described by SDE (18) (19) and (20) in [17]. In the case of the backward-in-time approach, quasi-particle starts its way from the registration (target) position (in all simulations that were presented in the article, this position was taken to be $1 \mathrm{AU}$ ) and propagate to the heliosphere boundary. Unlike from forward-in-time approach, in backward-in-time case particle at every moment of time gain momentum by values evaluated by Equation (19) in [17].

\section{Statistical Error for Selected Energies, Backward-in-Time Method}

The situation in the backward-in-time method is more straightforward than the forward-in-time approach [19]. Every simulated quasiparticle is registered in the backwardin-time approach. It means that every quasiparticle traced back in time from the injection position in the heliosphere eventually reaches the border of the heliosphere.

We simulated ten separate sets of one million quasiparticles with energy $5 \mathrm{GeV}$ at $1 \mathrm{AU}$ to illustrate how an error in the backward in time method change with a number of injected particles. Every trajectory was injected at $1 \mathrm{AU}$ with kinetic energy $5 \mathrm{GeV}$ and propagated through the heliosphere backward-in time till the trajectory cross heliosphere border. The kinetic energy of the quasiparticle at the heliosphere border was recorded. Then, having a big set of simulated trajectories, we evaluated intensity first from a small part of quasiparticle trajectories and then again from a bigger part, and again from an even bigger part, repeating this process for incrementally bigger sets. Specifically, we start with an intensity at $1 \mathrm{AU}$ evaluated from 1000 quasiparticle trajectories (taking the first 1000 quasiparticles from a set of a million simulated quasiparticles), then we evaluated intensity for 2000 quasiparticles (taking the first 2000 simulated quasiparticles), then for 3000 quasiparticles, etc., with step one being 1000 quasiparticles, till one million quasiparticles. We repeat the same procedure for all ten sets with one million quasiparticles. By this, we want to show an example of how evaluated intensity changes with an increasing number of used quasiparticles. Figure 1 shows how evaluated intensity of $5 \mathrm{GeV}$ particles at $1 \mathrm{AU}$ changes with a number of used quasiparticles. Results for every simulated set of million quasiparticles is marked by different collor. The convergence of evaluated intensities with an increasing number of used quasiparticles is clearly seen. The intensity in the figure is normalized to one (i.e., divided by mean intensity for all 10 million quasiparticles). 


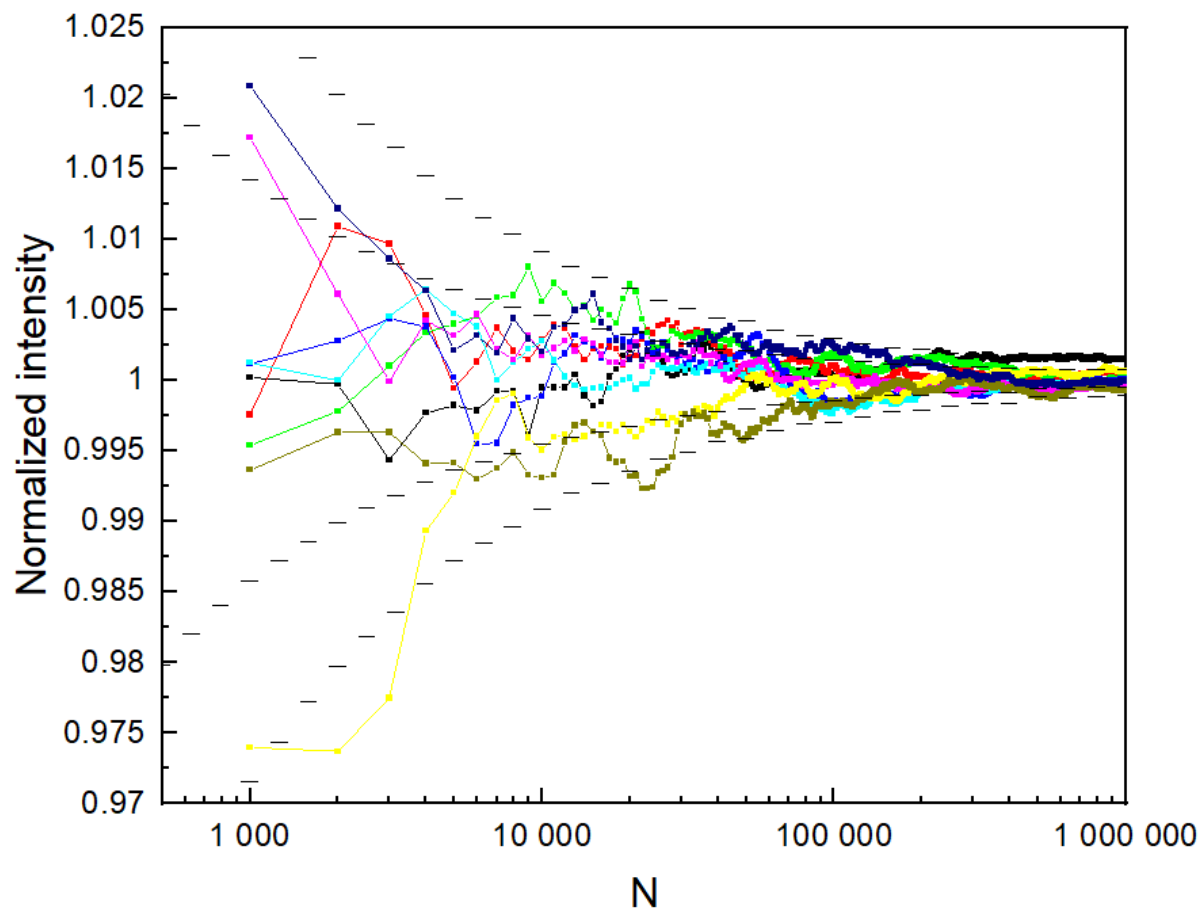

Figure 1. Dependence of intensity on number of simulated quasiparticles for ten separate set of quasiparticles signed by different colors (see details in text).

To show statistical error, we divided an obtained set of ten million quasiparticles to subsets with $N_{s}$ quasiparticles. For example, for $N_{s}=1000$ we get 10,000 subsets (from 10 million simulated quasiparticles). From every subset with $N_{s}=1000$ quasiparticles we evaluate an average value of intensity $I_{N s}$. Resulted 10,000 values of $I_{N} s$ has normal distribution as is presented in Figure 2. Red squares shows evaluated histogram of $I_{N s}$ distribution. Every subset of $N_{s}$ quasiparticles could be considered as random sampling from whole set, because every evaluation of quasiparticles trajectories is independent from each other evaluations.

Normal distribution appears here due to the central limit theorem as a result of averaging intensities of $N_{S}$ quasiparticles in subsets. Intensities of ten million simulated quasiparticles do not have a normal distribution. Only averaged values have a normal distribution. We evaluated mean $\mu_{N s}$ and standard deviation $\sigma_{N s}$ for a set of 10,000 values of $I_{N s}$ to describe obtained normal distribution. Resulted probability distribution function curve (red line in the figure) evaluated from $\mu_{N s}$ and $\sigma_{N s}$, as expected, nicely fit histogram of distribution. Standard deviation $\sigma_{N s}$ for this example, for $N_{s}=1000$ is 0.014 in $\mu$ units i.e., 1.4 percent from $\mu$ value. $\pm 1 \sigma$ range is shown in the figure. This is an example for input model parameters $K_{0}$ and $V$ set to approximate AMS-02 measurements in a period of May 2011. General dependency of a statistical error on $K_{0}$ and $V$ is presented by us later in the text.

Horizontal dash black lines in Figure 1 show $\mu \pm n \sigma$ for $n=1$, 2. As expected, almost all results in example in Figure 1 are in $2 \sigma$ range around $\mu$ value (because we have normal distribution here, $95.45 \%$ of all cases should be in $\mu \pm 2 \sigma$ ).

Figure 2 shows also evaluation for $N_{s}=100$ (here we have $10^{5}$ values of $I_{N s}$ ) shown in blue color. As expected distribution for $N_{s}=100$ is wider, and has value $\sigma_{N s} 0.045$ in $\mu$ units. The dependency of $\sigma_{N s}$ at $N_{s}$ for sets with normal distribution should have power law shape with slope $-1 / 2$. To show it we evaluated a $\sigma_{N s}$ for set of different $N_{s}$ with logarithmical step. In Figure 3. we show dependency of $\sigma$ on $N_{s}$ for evaluation of intensities for two energies Tkin equal $5 \mathrm{GeV}$ and $1 \mathrm{GeV}$. Evaluated $\sigma$ values in the percent of $\mu$ units are presented by points. Linear fit of both dependencies in log-log scale has slope with value very close to expected value $-1 / 2$, i.e., $\sigma \sim N_{s}^{-1 / 2}$. $\sigma$ values evaluated for 
same $N_{S}$ have higher values for lower energies. To reach same statistical error for lower energies, higher number of simulated particles, i.e., higher $N_{s}$ is needed. To investigate how $\sigma$ depend on energy for wider range of energies we simulated $10^{5}$ quasiparticles for every energy and average them by groups of 100 quasiparticles $\left(N_{s}=100\right)$ to evaluate $\sigma$ for every energy from one thousand intensities. We realize simulations for energies from $1 \mathrm{GeV}$ to $100 \mathrm{GeV}$ for three combinations of input values of diffusion coefficient $K_{0}=3 \times 10^{18}$, $5 \times 10^{18}, 7 \times 10^{18} \mathrm{~m}^{2} / \mathrm{s}$ and solar wind velocity $V=400 \mathrm{~km} / \mathrm{s}$. Results are presented in Figure 4 where $\sigma$ values in the percent $\mu$ units are shown in red color for $K_{0}=3 \times 10^{18} \mathrm{~m}^{2} / \mathrm{s}$, for $K_{0}=5 \times 10^{18} \mathrm{~m}^{2} / \mathrm{s}$ in black color and for $K_{0}=7 \times 10^{18} \mathrm{~m}^{2} / \mathrm{s}$ in blue color. Range of diffusion values was selected to cover usual values used to simulate measured spectra at 1 AU by 1D SDE models. The dependency, where $\sigma$ is bigger for smaller energies presented in Figure 3 is more clearly visible in Figure 4. One could notice that with smaller values of diffusion coefficients are $\sigma$ values bigger.

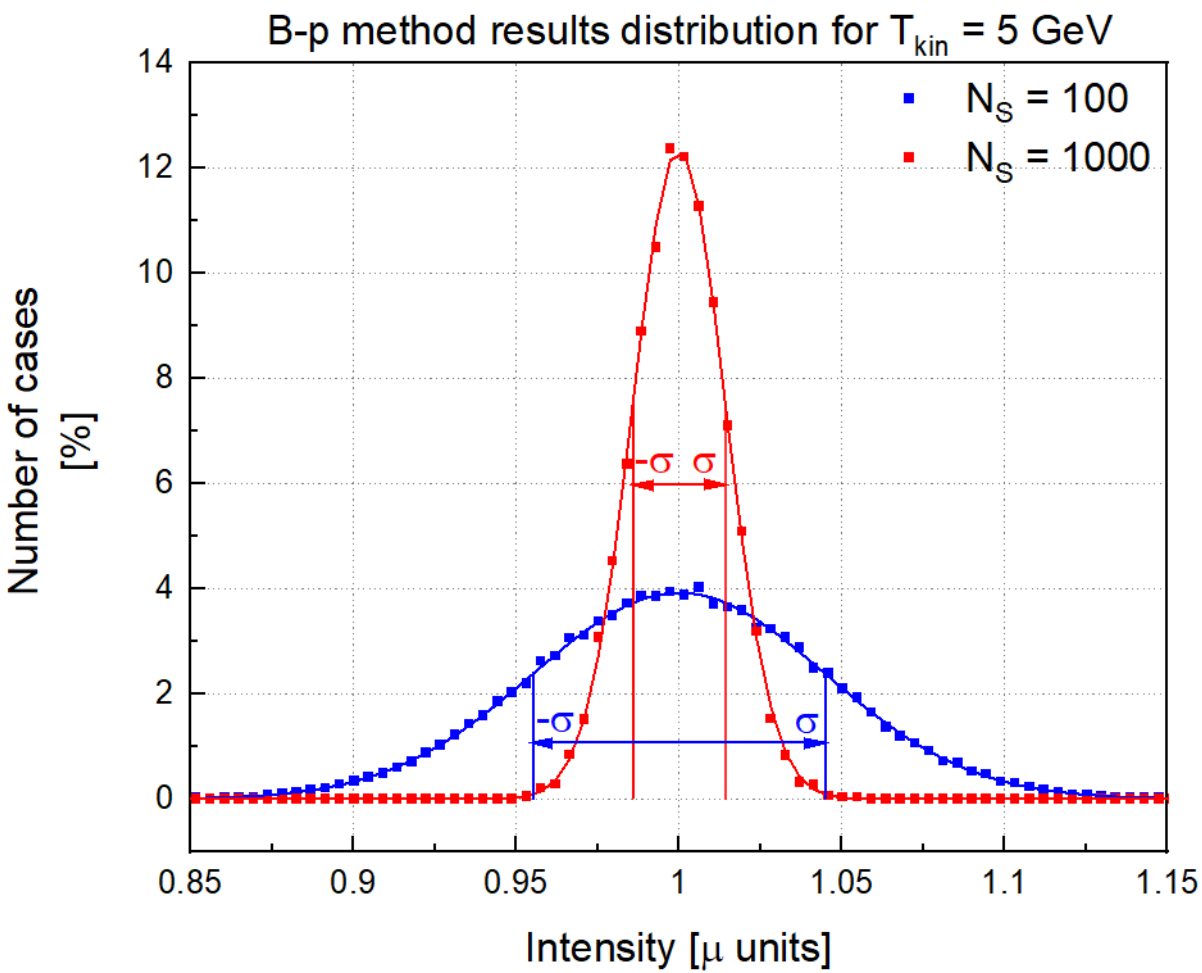

Figure 2. Comparison of $I_{N_{s}}$ values histogram with probability distribution function with $\mu_{N s}$ and $\sigma_{N s}$ of histogram. Red points sign case for $N_{s}=1000$, blue for $N_{s}=100$. Ranges $\pm 1 \sigma$ are shown for both cases.

We could notice, that for example for $1 \mathrm{GeV}$ particles simulation with 100 quasiparticles $\left(N_{s}=100\right)$ has $\sigma$ value close to 3 percent in $\mu$ units for $K_{0}=3 \times 10^{18} \mathrm{~m}^{2} / \mathrm{s}$ (signed by red in figure) and 6 percent for $K_{0}=7 \times 10^{18} \mathrm{~m}^{2} / \mathrm{s}$ (signed by blue color in figure). For $10 \mathrm{GeV}$ particles, we get from simulation with 100 quasiparticles $\sigma$ close to 2 percent for $K_{0}=3 \times 10^{18} \mathrm{~m}^{2} / \mathrm{s}$ and close to 1 percent for $K_{0}=7 \times 10^{18} \mathrm{~m}^{2} / \mathrm{s}$.

How exactly depend $\sigma$ on diffusion coefficient, which could be seen in Figure 5 where we show dependencies for selected energies 1, 3, 10, 30 and $100 \mathrm{GeV}$. Dependencies have power law shape with slopes very close to value -1.0 for higher energies (over the few tens of $\mathrm{GeV}$ ), i.e., $\sigma \sim K_{0}^{-1.0}$. For smaller energies, in a region with significant solar modulation, slope value decreases, for example, for energy $1 \mathrm{GeV}$ is close to -0.8 , i.e., $\sigma \sim K_{0}^{-0.8}$. 


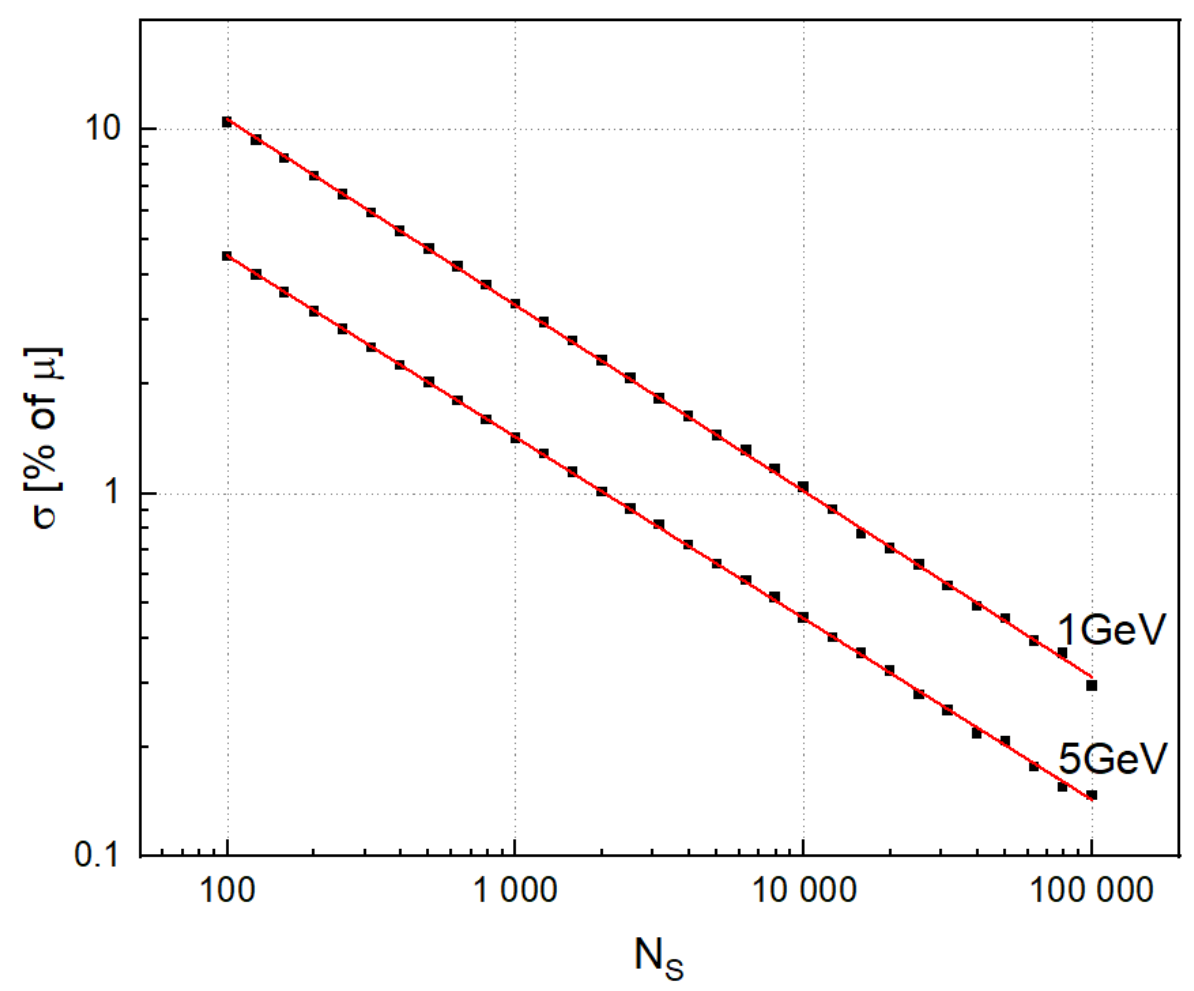

Figure 3. Dependency of $\sigma$ on $N_{S}$ for energies Tkin equal $5 \mathrm{GeV}$ and $1 \mathrm{GeV}$ (for details see text).

With knowledge that $\sigma$ has power law dependency on $N_{S}$ with slope $-1 / 2$ (consequence of central limit theorem and resulted normal distributions of averaged intensities, then $\sigma$ is standard error of the mean) and with simulation for one $N_{S}$ we could evaluate $\sigma$ values for other $N_{S}$ values. Thus, we could evaluate how many quasiparticles we need to simulate and reach statistical error at a level of 1 percent. In other words, to reach $\sigma$ equals 1 percent of mean value $\mu$. Results for three values of diffusion coefficient $K_{0}=3 \times 10^{18}, 5 \times 10^{18}, 7 \times 10^{18} \mathrm{~m}^{2} / \mathrm{s}$, solar wind velocity $V=400 \mathrm{~km} / \mathrm{s}$ and five selected energies $T_{k i n}=1,5,10,20,50 \mathrm{GeV}$ are shown in Table 1 .

We could notice range of needed simulation from a few to a couple of thousands. For diffusion coefficient $K_{0}=3 \times 10^{18} \mathrm{~m}^{2} / \mathrm{s}$, we need only 12 particles to reach $\sigma$ equal 1 percent in $\mu$ units for particles with energy $50 \mathrm{GeV}$. However, for energy $1 \mathrm{GeV}$ we need to simulate almost four thousand particles to reach the same $\sigma$ value. While for diffusion coefficient $K_{0}=7 \times 10^{18} \mathrm{~m}^{2} / \mathrm{s}$ for $50 \mathrm{GeV}$ we need only two particles to have $\sigma$ at one percent level, and for $1 \mathrm{GeV}$ we need almost one thousand of simulated particles. As a consequence, we could set simple simulation strategies to keep statistical error of simulation limited to $\sigma$ at a 1 percent level, i.e., get evaluated intensities for all energies with statistical errors $\sigma$ smaller than one percent. For the case presented in Table 1, i.e., for solar wind velocity $400 \mathrm{~km} / \mathrm{s}$ it could be, for example, a safe strategy where we simulate for every energy $10^{4} / T_{\text {kin }}$ quasiparticles, where $T_{\text {kin }}$ is in $\mathrm{GeV}$.

Using this strategy we reach for all energies $\sigma<1 \%$, thus we will have 68 percent of results in $\pm 1 \%$ of $\mu$ and 95 percent of results in $\pm 2 \%$ of $\mu$. Here, $\mu$ practically means value which we get for simulations with very high numbers of quasiparticles. 
Table 1. Number of injected simulations needed to reach $\sigma$ value equaling 1 percent, in units of percent of $\mu$, for different energy bins $(1,5,10,20,50 \mathrm{GeV})$. Evaluation for solar wind velocity $400 \mathrm{~km} / \mathrm{s}$.

\begin{tabular}{ccc}
\hline & $\sigma=\mathbf{1}[\%$ of $\mu$ units] & \\
\hline$K_{\mathbf{0}}$ & Energy [GeV] & $\boldsymbol{N}$ \\
\hline \multirow{3}{*}{$3 \times 10^{18} \mathrm{~m}^{2} \mathrm{~s}^{-1}$} & 1 & 3785.0 \\
& 5 & 585.7 \\
& 20 & 214.6 \\
& 50 & 67.8 \\
& 1 & 12.2 \\
\hline \multirow{2}{*}{$5 \times 10^{18} \mathrm{~m}^{2} \mathrm{~s}^{-1}$} & 5 & 1719.9 \\
& 10 & 250.4 \\
& 20 & 82.5 \\
& 50 & 24.2 \\
& 1 & 4.9 \\
\hline \multirow{2}{*}{$7 \times 10^{18} \mathrm{~m}^{2} \mathrm{~s}^{-1}$} & 5 & 968.4 \\
& 10 & 127.0 \\
& 20 & 44.2 \\
& 50 & 12.9 \\
\hline
\end{tabular}

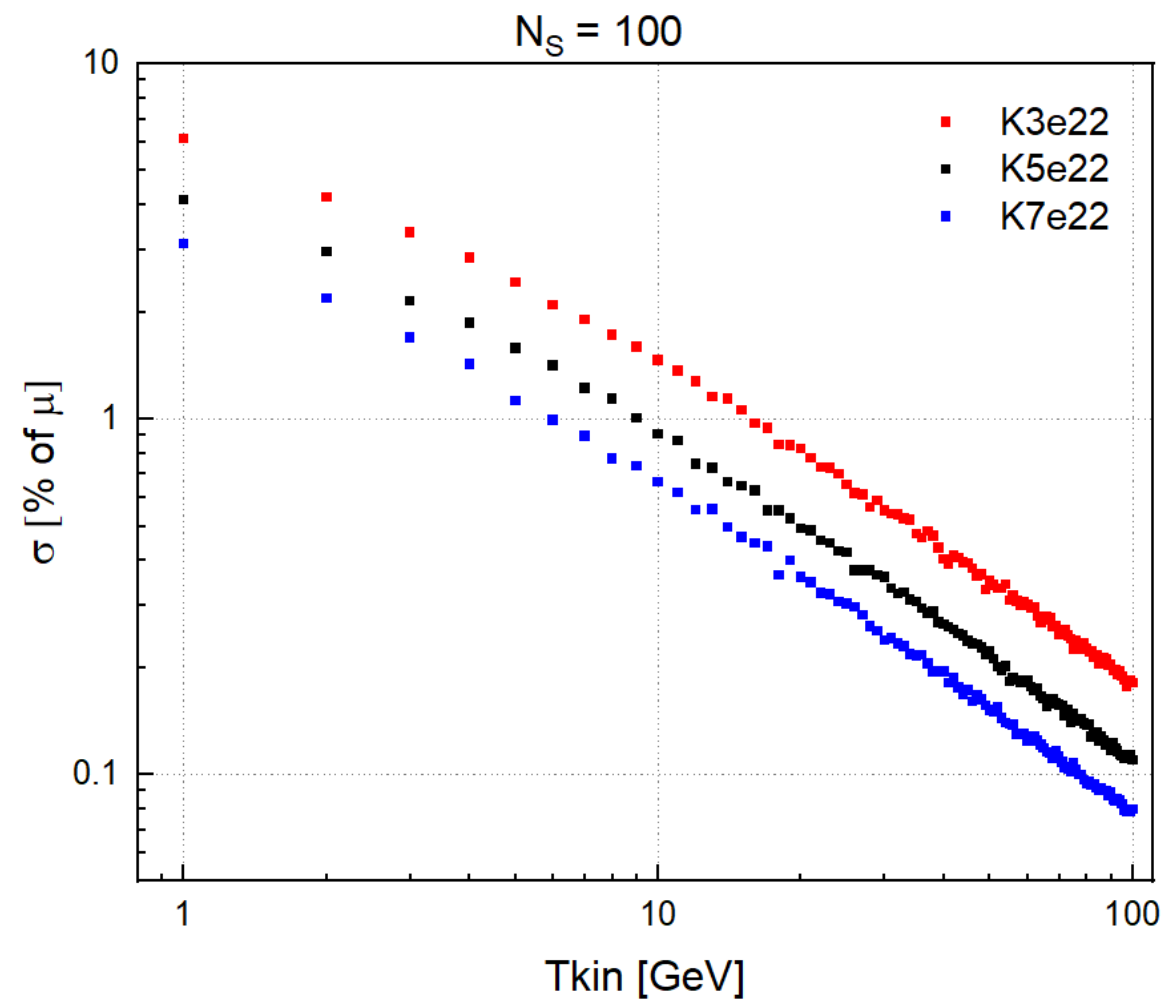

Figure 4. $\sigma$ for diffusion coefficients $K_{0}=3 \times 10^{18}, 5 \times 10^{18}, 7 \times 10^{18} \mathrm{~m}^{2} / \mathrm{s}$ and $V=400 \mathrm{~km} / \mathrm{s}$ evaluated for energies from $1 \mathrm{GeV}$ to $100 \mathrm{GeV}$.

Both input parameters, diffusion coefficient and solar wind velocity have influence to statistical error expressed by $\sigma$. Dependency of $\sigma$ on solar wind velocity is presented in Figure 6 . In this figure we show evaluation for solar wind velocities $V=300,400,500 \mathrm{~km} / \mathrm{s}$ and diffusion coefficient $K_{0}=5 \times 10^{18} \mathrm{~m}^{2} / \mathrm{s}$. Evaluation of $\sigma$ was done for the same parameter $N_{S}=100$ and same number of simulated quasipatricles, $10^{5}$, for every energy from range of energies from $1 \mathrm{GeV}$ to $100 \mathrm{GeV}$ with the $1 \mathrm{GeV}$ energy step. $\sigma$ increases with 
higher values of solar wind speed. Statistical error is higher, when less particles reach the position at $1 \mathrm{AU}$. Interestingly, dependence of $\sigma$ on solar wind velocity at high energies has slope closely equal to 1.0, i.e., $\sigma \sim V^{1.0}$ as could be seen in Figure 7. For smaller energies, where solar modulation is bigger, for example, for energy $1 \mathrm{Gev}$ slope is close to 0.8 , i.e., $\sigma \sim V^{0.8}$.

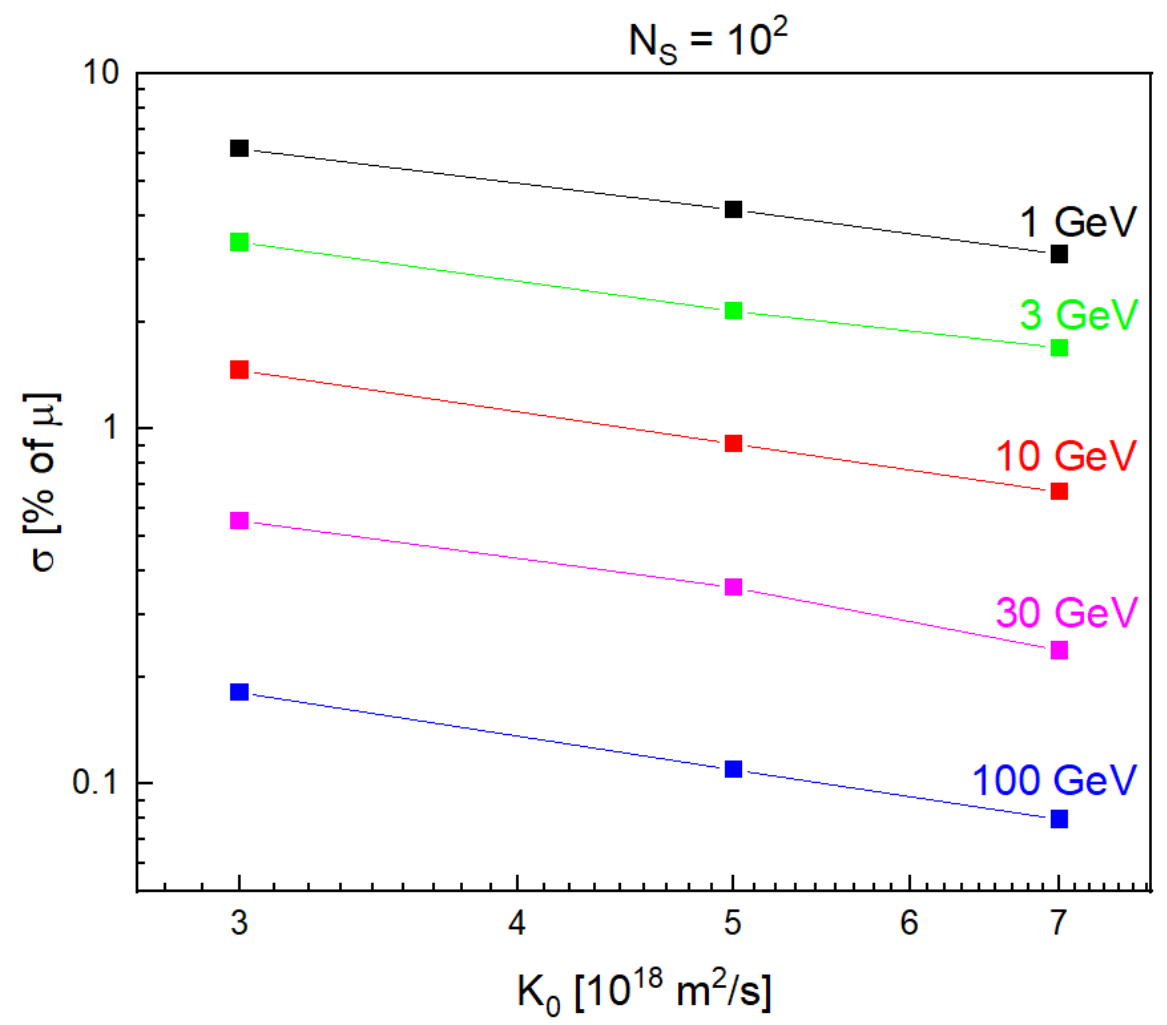

Figure 5. $\sigma$ for diffusion coefficients $K_{0}=3 \times 10^{18}, 5 \times 10^{18}, 7 \times 10^{18} \mathrm{~m}^{2} / \mathrm{s}$ and $V=400 \mathrm{~km} / \mathrm{s}$ evaluated for energies $1,3,10,30$ and $100 \mathrm{GeV}$.

As we present previously for Table 1, where we did it for different diffusion coefficients and fixed solar wind velocity, we could evaluate $\sigma$ values to get $N_{S}$ values to reach statistical error at level of 1 percent of $\mu$ for fixed value of diffusion coefficient and varied $V$ value. Here we use simulations with $K_{0}=5 \times 10^{18} \mathrm{~m}^{2} / \mathrm{s}$ and vary solar wind velocities with $V=300,400,500 \mathrm{~km} / \mathrm{s}$. Used range of solar wind velocities is usual range of averaged values from periods close to one Bartols rotation, for which experimental results are usually published. Results are presented in Table 2 . One could notice that for solar wind velocity $V=300 \mathrm{~km} / \mathrm{s}$ for $50 \mathrm{GeV}$ we need only three particles to have $\sigma$ at one percent level, and for $1 \mathrm{GeV}$ we need more than one thousand of simulated particles. For solar wind velocity $V=500 \mathrm{~km} / \mathrm{s}$ for $50 \mathrm{GeV}$ we need eight particles to have $\sigma$ at one percent level, and for $1 \mathrm{GeV}$ we need two and half thousand of simulated particles. With faster solar wind we need more simulated quasiparticles to reach the same statistical error. 
Table 2. Number of injected simulations needed to reach $\sigma$ value equal 1 percent, in units of percent of $\mu$, for different energy bins $(1,5,10,20,50 \mathrm{GeV})$. Evaluated with diffusion coefficient $K_{0}=5 \times 10^{18} \mathrm{~m}^{2} / \mathrm{s}$.

\begin{tabular}{ccc}
\hline & $\sigma=\mathbf{1}[\%$ of $\mu$ units $]$ & \\
\hline SW Velocity & Energy [GeV] & $N$ \\
\hline \multirow{3}{*}{$V_{s w}=300 \mathrm{~km} \mathrm{~s}^{-1}$} & 1 & 1063.5 \\
& 5 & 162.6 \\
& 10 & 48.0 \\
& 20 & 14.6 \\
$V_{s w}=400 \mathrm{~km} \mathrm{~s}^{-1}$ & 50 & 2.6 \\
\hline & 1 & 1719.9 \\
& 5 & 250.4 \\
& 10 & 82.5 \\
$V_{s w}=500 \mathrm{~km} \mathrm{~s}^{-1}$ & 20 & 24.2 \\
& 50 & 4.9 \\
\hline & 1 & 2419.2 \\
& 10 & 369.5 \\
& 20 & 136.6 \\
& 50 & 38.6 \\
\end{tabular}

Safe simulation strategy for varied diffusion values and $V$ fixed at $400 \mathrm{~km} / \mathrm{s}$ mentioned previously here, with $10^{4} / T_{\text {kin }}$ particles simulated for energy $T_{k i n}$ in $\mathrm{GeV}$, stay still valid when we vary solar wind velocity. The combination of solar wind velocity and diffusion coefficient with the biggest statistical error is $K_{0}=3 \times 10^{18} \mathrm{~m}^{2} / \mathrm{s}$ and $V=500 \mathrm{~km} / \mathrm{s}$. For this combination, we need 4895 simulated quasiparticles with kinetic energy $1 \mathrm{GeV}$ to reach $\sigma$ at level one percent from $\mu$. Thus strategy with $10^{4} / T_{\text {kin }}$ particles simulated for energy $T_{k i n}$ in $\mathrm{GeV}$ could be considered as safe.

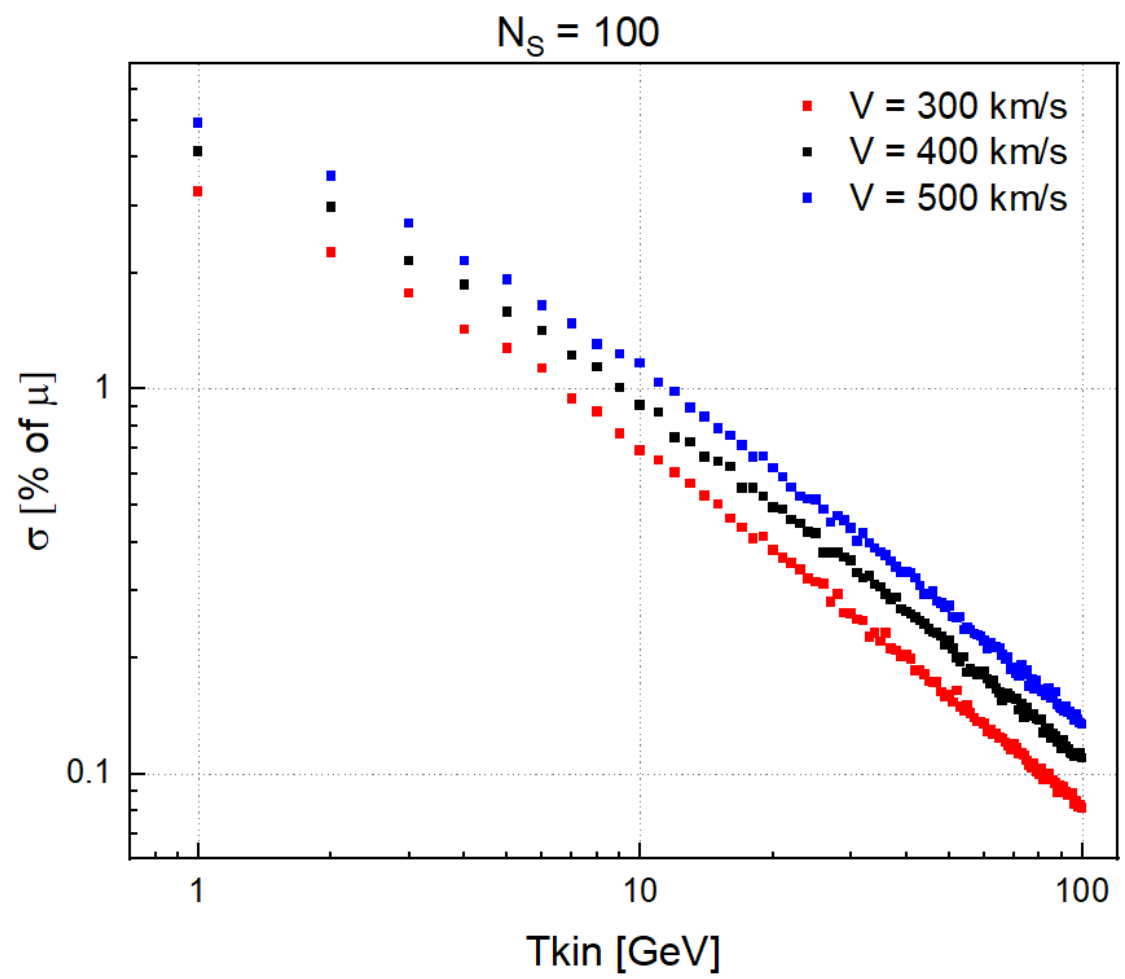

Figure 6. $\sigma$ for solar wind velocity $V=300,400,500 \mathrm{~km} / \mathrm{s}$ and diffusion coefficient $K_{0}=5 \times 10^{18} \mathrm{~m}^{2} / \mathrm{s}$ evaluated for energies from $1 \mathrm{GeV}$ to $100 \mathrm{GeV}$. 


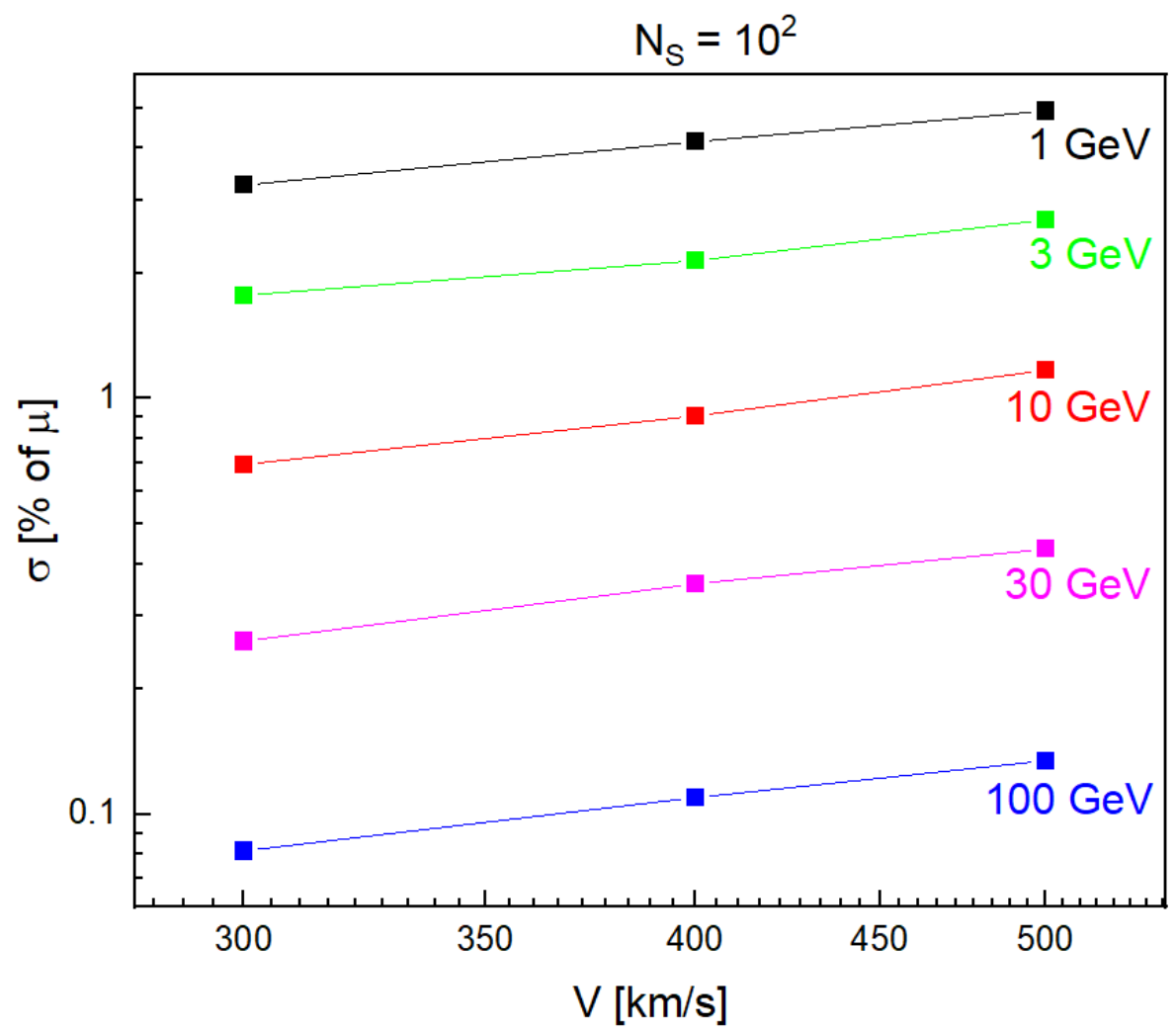

Figure 7. $\sigma$ for solar wind velocity $V=300,400,500 \mathrm{~km} / \mathrm{s}$ and diffusion coefficient $K_{0}=5 \times 10^{18} \mathrm{~m}^{2} / \mathrm{s}$ evaluated for energies $1,3,10,30$ and $100 \mathrm{GeV}$.

The turbulence spectra have also an effect on statistical error in backward-in-time SDE method. Diffusion coefficient as a function of particle rigidity presented in the previous part depends on rigidity linearly $\left(K=K_{0} \beta P^{1.0}\right)$. However, dependency on rigidity could have a different slope $K=K_{0} \beta P^{\alpha}$ with $\alpha$ bigger or smaller than one. We tested influence of different values of $\alpha$ to statistical error for range from $\alpha=0.6$ to $\alpha=1.4$. To test influence of these $\alpha$ values to statistical error we used the same method as before and evaluate $\sigma$ for $N_{S}=100, K_{0}=5 \times 10^{18} \mathrm{~m}^{2} / \mathrm{s}$ and $V=400 \mathrm{~km} / \mathrm{s}$. Results for the range of kinetic energies from $1 \mathrm{Gev}$ to $100 \mathrm{Gev}$ are shown in Figure 8. As inspection of the figure shows, with smaller $\alpha$ values we have bigger statistical error. The difference between $\sigma$ values for different $\alpha$ raise with kinetic energy i.e., is smallest for small energies. We used results presented in Figure 8 to evaluate number of simulated quasiparticles needed to reach statistical error $\sigma$ equaling 1 percent from intensity mean value $\mu$ at different energies. Results for selected energies are shown in Table 3. for two situations. The first one with typical values of the diffusion coefficient and solar wind velocity, for $K_{0}=5 \times 10^{18} \mathrm{~m}^{2} / \mathrm{s} \& V=400 \mathrm{~km} / \mathrm{s}$ which we call average situation. The second one for combination of input parameters with maximal statistical error, is for $K_{0}=3 \times 10^{18} \mathrm{~m}^{2} / \mathrm{s} \& V=500 \mathrm{~km} / \mathrm{s}$. In a situation, with a maximum statistical error, we need for kinetic energy $1 \mathrm{GeV} \sim$ six thousand simulated quasiparticles to reach $\sigma$ at level 1 percent of $\mu$. Recommended strategy with $10^{4} / T_{\text {kin }}$ particles simulated for energy $T_{k i n}$ in $\mathrm{GeV}$ is still safe for parameters evaluated in Table 3 . To compare our suggested strategy with other authors we could reference only [25] and the current article [26]. The first author generally mentions that typically a few thousand stochastic processes can yield a result with better than $3 \%$ error, without specifying energies or ranges of input parameters used for modeling. In the second authors, they found that the generation of $N \sim 2 \times 10^{3}$ pseudoparticles for each energy bin is sufficient for being not dominated by SDE-related uncertainties. Which is generally consistent with our recommended safe simulation strategy. 
Table 3. Number of injected simulations needed to reach $\sigma$ value equal 1 percent, in units of percent of $\mu$, for different energy bins $(1,5,10,20,50 \mathrm{GeV})$. Evaluated with diffusion coefficient $K_{0}=5 \times 10^{18} \mathrm{~m}^{2} / \mathrm{s} \&$ solar wind velocity $V=400 \mathrm{~km} / \mathrm{s}$ (third column, named average situation) and $K_{0}=3 \times 10^{18} \mathrm{~m}^{2} / \mathrm{s} \& V=500 \mathrm{~km} / \mathrm{s}$ (fourth column, marked as situation with maximal statistical error) for different $\alpha$ values.

\begin{tabular}{cccc}
\hline \multicolumn{4}{c}{$\sigma=\mathbf{1}[\%$ of $\boldsymbol{\mu}$ units] } \\
\hline$\alpha$ & Energy [GeV] & $\boldsymbol{N}$, Avg. Situation & $\boldsymbol{N}$, Maximum Error \\
\hline \multirow{4}{*}{$\alpha=0.6$} & 1 & 2837.9 & 6368 \\
& 5 & 892.2 & 2102.9 \\
& 10 & 464.2 & 1095.5 \\
& 20 & 252.46 & 569.4 \\
$\alpha=1.0$ & 50 & 89.0 & 241.9 \\
\hline & 1 & 1719.9 & 4895 \\
& 5 & 250.4 & 853 \\
& 10 & 82.5 & 320.3 \\
& 20 & 24.2 & 96 \\
& 50 & 4.9 & 18.2 \\
\hline \multirow{4}{*}{$\alpha=1.4$} & 1 & 1038.41 & 1821.4 \\
& 5 & 69.2 & 34.6 \\
& 10 & 14.2 & 6.7 \\
& 20 & 2.3 & 0.5 \\
\hline
\end{tabular}

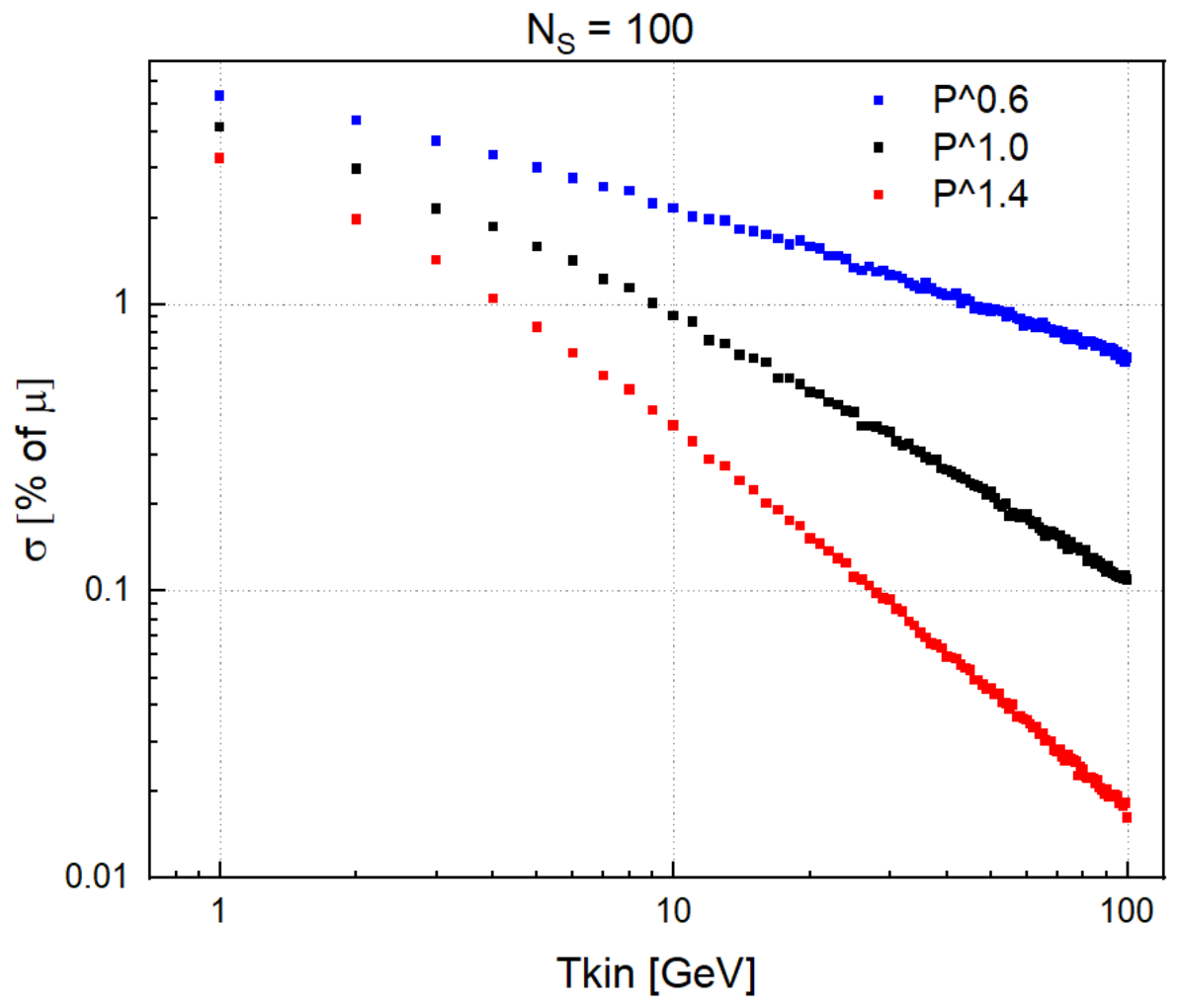

Figure 8. $\sigma$ for solar wind velocity $V=400 \mathrm{~km} / \mathrm{s}$, diffusion coefficient $K_{0}=5 \times 10^{18} \mathrm{~m}^{2} / \mathrm{s}$ evaluated for range of kinetic energies between 1 to $100 \mathrm{GeV}$ for $\alpha=0.6,1.0,1.4$.

Let us note, that all previously presented results are independent of the time step of simulation $\Delta t$ value when keeping condition for dominancy of diffusion over convection valid. The results were tested in simulations with different time steps and the results were very similar. 


\section{Conclusions}

The statistical error of the SDE backward-in-time 1D approach of Parker's equation solution was analyzed. We show that intensities evaluated with this approach have, due to central limit theorem, normal distribution. We present that standard deviation $\sigma$ of intensities normal distribution depend on a number of averaged intensities, i.e., on a number of simulated quasiparticles, with power law dependency with slope $-1 / 2$. Dependency of $\sigma$ on input parameters of the simulation, diffusion coefficient $K_{0}$, and solar wind speed $V$ was studied. Dependency of $\sigma$ on $K_{0}$ has power law shape with slope -1.0 at high energies and higher, but close to -1.0 values for lower energies from the region where solar modulation is maximal, i.e., for $1 \mathrm{GeV}$ slope close to -0.8 . Dependency of $\sigma$ on $V$ has power law shape with slope 1.0 at high energies and smaller, but close to 1.0 values for lower energies, i.e., for $1 \mathrm{GeV}$ slope close to 0.8 . Based on obtained results, the safe simulation strategy for spectra evaluation at $1 \mathrm{AU}$ was suggested. Simulate for every energy $10^{4} / T_{k i n}$ quasiparticles $\left(T_{k i n}\right.$ in $\left.\mathrm{GeV}\right)$. This strategy leads to results where we have for every energy in the evaluated spectrum statistical error $\sigma$ smaller than 1 percent of $\mu$ value for normal distribution of intensities for selected energy.

Author Contributions: Conceptualization, V.M. and P.B.; Methodology, V.M. and P.B.; Software, V.M. and P.B.; Validation, V.M. and P.B.; Formal analysis, V.M. and P.B.; Investigation, V.M. and P.B.; Writing and editing, V.M. and P.B. All authors have read and agreed to the published version of the manuscript.

Funding: This work was partially supported by the Slovak Academy of Sciences, grant MVTS JEM-EUSO as well as by VEGA grant agency project 2/0077/20.

Institutional Review Board Statement: Not applicable.

Informed Consent Statement: Not applicable.

Data Availability Statement: Not applicable.

Acknowledgments: Authors are grateful to the reviewers for constructive suggestions and for proposed corrections to improve the paper.

Conflicts of Interest: The author declares no conflict of interest.

\section{References}

1. Parker, E. The passage of energetic charged particles through interplanetary space. Planet. Space Sci. 1965, 13, 9-49. [CrossRef]

2. Jokipii, J.R.; Levy, E.H.; Hubbard, W.B. Effects of particle drift on cosmic-ray transport. I: General properties, application to solar modulation. Astrophys. J. Lett. 1977, 213, 861-868. [CrossRef]

3. Chandrasekhar, S. Stochastic Problems in Physics and Astronomy. Rev. Mod. Phys. 1943, 15, 1-89. [CrossRef]

4. Kota, J. Energy loss in the solar system and modulation of cosmic radiation. In Proceedings of the 15th International Cosmic Ray Conference, Plovdiv, Blgaria, 13-26 August 1977; pp. 186-191.

5. Zhang, M. A Markov Stochastic Process Theory of Cosmic-Ray Modulation. Astrophys. J. Lett. 1999, 513, 409-420. [CrossRef]

6. Florinski, V.; Pogorelov, N.V. Four-dimensional transport of galactic cosmic rays in the outer heliosphere and heliosheath. Astrophys. J. Lett. 2009, 701, 642-651. [CrossRef]

7. Strauss, D.T.; Potgieter, M.; Kopp, A.; Büsching, I. On the propagation times and energy losses of cosmic rays in the heliosphere J. Geophys. Res. Earth Surf. 2011, 116. [CrossRef]

8. Strauss, R.D.; Potgieter, M.S.; Büsching, I.; Kopp, A. Modelling heliospheric current sheet drift in stochastic cosmic ray transport models. Astrophys. Space Sci. 2012, 339, 223-236. [CrossRef]

9. Effenberger, F.; Fichtner, H.; Scherer, K.; Büsching, I. Anisotropic diffusion of Galactic cosmic ray protons and their steady-state azimuthal distribution. Astron. Astrophys. 2012, 547, A120. [CrossRef]

10. Strauss, D.T.; Potgieter, M.; Ferreira, S.; Fichtner, H.; Scherer, K. Cosmic ray modulation beyond the heliopause: A hybrid modeling approach. Astrophys. J. 2013, 765, L18. [CrossRef]

11. Zhao, L.-L.; Qin, G.; Zhang, M.; Heber, B. Modulation of galactic cosmic rays during the unusual solar minimum between cycles 23 and 24. J. Geophys. Res. Space Phys. 2014, 119, 1493-1506. [CrossRef]

12. Engelbrecht, N.E.; Burger, R. Sensitivity of cosmic-ray proton spectra to the low-wavenumber behavior of the $2 \mathrm{~d}$ turbulence power spectrum. Astrophys. J. Lett. 2015, 814, 152. [CrossRef]

13. Moloto, K.D.; Engelbrecht, N.E.; Burger, R. A Simplified Ab Initio Cosmic-ray Modulation Model with Simulated Time Dependence and Predictive Capability. Astrophys. J. Lett. 2018, 859, 107. [CrossRef] 
14. Shen, Z.-N.; Qin, G.; Zuo, P.; Wei, F. Modulation of Galactic Cosmic Rays from Helium to Nickel in the Inner Heliosphere. Astrophys. J. 2019, 887, 132. [CrossRef]

15. Du Toit Strauss, R.; Effenberger, F. A Hitch-hiker's Guide to Stochastic Differential Equations. Solution Methods for Energetic Particle Transport in Space Physics and Astrophysics. Space Sci. Rev. 2017, 212, 151-192. [CrossRef]

16. Kopp, A.; Büsching, I.; Strauss, R.; Potgieter, M. A stochastic differential equation code for multidimensional Fokker-Planck type problems. Comput. Phys. Commun. 2012, 183, 530-542. [CrossRef]

17. Bobik, P.; Boschini, M.J.; Della Torre, S.; Gervasi, M.; Grandi, D.; La Vacca, G.; Pensotti, S.; Putis, M.; Rancoita, P.G.; Rozza, D.; et al. On the forward-backward-in-time approach for Monte Carlo solution of Parker's transport equation: One-dimensional case. J. Geophys. Res. Space Phys. 2016, 121, 3920-3930. [CrossRef]

18. Wawrzynczak, A.; Modzelewska, R.; Gil, A. Algorithms for Forward and Backward Solution of the Fokker-Planck Equation in the Heliospheric Transport of Cosmic Rays. In Parallel Processing and Applied Mathematics, PPAM 2017; Lecture Notes in Computer Science; Springer: Cham, Switzerland, 2018; Volume 10777, pp. 14-23. [CrossRef]

19. Mykhailenko, V.; Bobik, P. Statistical error for cosmic rays modulation evaluation by $1 \mathrm{D}$ and 2D models. In Proceedings of the 37th International Cosmic Ray Conference (ICRC2021), Berlin, Germany, 12-23 July 2021; Volume 395, p. 1325. [CrossRef]

20. Moloto, K.; Engelbrecht, N.; Strauss, R.; Moeketsi, D.; Berg, J.V.D. Numerical integration of stochastic differential equations: A parallel cosmic ray modulation implementation on Africa's fastest computer. Adv. Space Res. 2018, 63, 626-639. [CrossRef]

21. Pei, C.; Bieber, J.W.; Burger, R.; Clem, J. A general time-dependent stochastic method for solving Parker's transport equation in spherical coordinates. J. Geophys. Res. Space Phys. 2010, 115. [CrossRef]

22. Yamada, Y.; Yanagita, S.; Yoshida, T. A stochastic view of the solar modulation phenomena of cosmic rays. Geophys. Res. Lett. 1998, 25, 2353-2356. [CrossRef]

23. Burger, R.A.; Moraal, H.; Webb, G.M. Drift theory of charged particles in electric and magnetic fields. Astrophys. Space Sci. 1985, 116, 107-129. [CrossRef]

24. Hattingh, M.; Burger, R. A new simulated wavy neutral sheet drift model. Adv. Space Res. 1995, 16, 213-216. [CrossRef]

25. Zhang, M. A Stochastic Differential Equation Approach to Cosmic Ray Transport. In Proceedings of the Numerical Modeling of Space Plasma Flows: Astronum 2007 ASP Conference Series, Paris, France, 10-15 June 2007; Volume 385, p. 63.

26. Fiandrini, E.; Tomassetti, N.; Bertucci, B.; Donnini, F.; Graziani, M.; Khiali, B.; Conde, A.R. Numerical modeling of cosmic rays in the heliosphere: Analysis of proton data from AMS-02 and PAMELA. Phys. Rev. D 2021, 104, 023012. [CrossRef] 